\title{
Rate of Anti-NMDA Receptor Encephalitis in Ovarian Teratomas
}

\author{
Jennifer H. Li ${ }^{1}$ Sarah S. Milla²,3 Grace Y. Gombolay,20
}

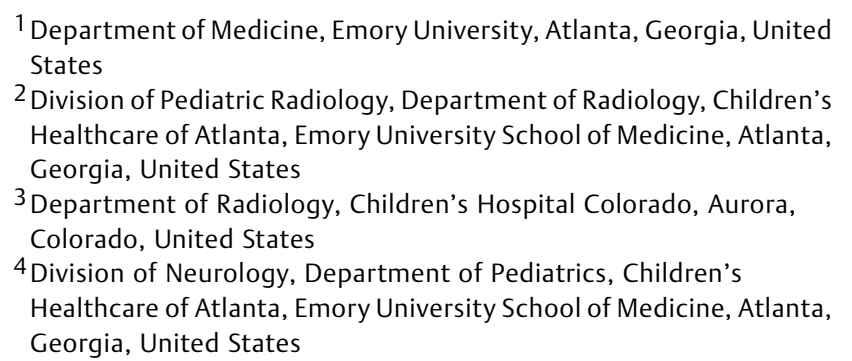

Address for correspondence Grace Gombolay, MD, 1400 Tullie Road NE, 8th Floor, Atlanta, Georgia, 30329, United States

(e-mail: ggombol@emory.edu).

Neuropediatrics 2022;53:133-135.

\begin{abstract}
Keywords

- autoimmune encephalitis

- anti-NMDA receptor encephalitis

- ovarian teratoma

- paraneoplastic

Background The rate of anti-N-methyl-D-aspartate receptor encephalitis (NMDARE) in ovarian teratomas is unknown. We aim to identify the prevalence of NMDARE as well as volumetric and histopathologic characteristics of ovarian teratomas in patients with versus without.

Methods We performed a retrospective cohort study to identify patients with confirmed ovarian teratomas and the characteristics of teratomas in NMDARE compared with non-NMDARE patients. Patients aged between 0 and 21 years with confirmed histopathological diagnosis of ovarian teratoma after resection were included. The rate of NMDARE in ovarian teratomas was identified. Moreover, volumes of ovarian teratomas and the frequency of neuronal glial elements on histopathology in NMDARE versus non-NMDARE patients were assessed.

Results Five out of one-hundred-and-sixty-three (3.07\%) patients with histopathology confirmed ovarian teratomas were diagnosed with NMDARE. Age was not different between the NMDARE (mean: 13.8 years, standard deviation: 3.9) and non-NMDARE groups (median: 14, interquartile range [IQR]: 5). Teratoma volumes from NMDARE patients were smaller than those of non-NMDARE patients (median $28.3 \mathrm{~cm}^{3}$ with IQR of 431.2 and median 182.8 with IQR of 635.0 , respectively). Both age and NMDARE diagnosis were statistically significant variables in the analysis of variance on a multiple linear regression model. Age $(p=0.013)$ had a positive correlation with teratoma size, whereas presence of NMDARE had a negative correlation $(p=0.008)$.

Conclusion The rate of NMDARE in ovarian teratomas is low and NMDARE patients have smaller teratomas than non-NMDARE. Further studies are needed to understand the timing of anti-NMDA receptor antibodies in teratomas and the development of NMDARE.
\end{abstract}

received

July 13, 2021

accepted after revision

October 4, 2021

published online

December 6, 2021 (c) 2021. Thieme. All rights reserved.

Georg Thieme Verlag KG,

Rüdigerstraße 14,

70469 Stuttgart, Germany
DOI https://doi.org/

10.1055/s-0041-1740352.

ISSN 0174-304X. 


\section{Introduction}

Anti-N-methyl-D-aspartate receptor encephalitis (NMDARE) is an autoimmune encephalitis with neuropsychiatric symptoms caused by anti-NMDA receptor immunoglobulin G antibodies. ${ }^{1}$ While a paraneoplastic syndrome is most commonly associated with ovarian teratomas, ${ }^{1-4}$ the NMDARE rate in ovarian teratomas is unknown. In this retrospective study, we determine the rate of NMDARE in ovarian teratomas and compare tumor sizes between NMDARE and nonNMDARE patients.

\section{Methods}

Institutional review board approval (STUDY00000209) was obtained for this study. Informed consent was waived. Patients were identified by the Montage database system of radiological imaging by the search terms "ovarian" and "teratoma or dermoid" at a single tertiary pediatric free-standing hospital between January 2011 and December 2019. Chart review was performed on 163 of 461 patients that had confirmed ovarian teratomas by resection and pathology. NMDARE was diagnosed if patients met criteria for confirmed NMDARE defined as having positive anti-NMDA receptor cerebrospinal fluid (CSF) antibodies and one or more of six neuropsychiatric symptoms: (1) abnormal psychiatric behavior/cognitive dysfunction; (2) speech dysfunction; (3) seizures; (4) movement disorder, dyskinesias, or rigidity/abnormal postures; (5)

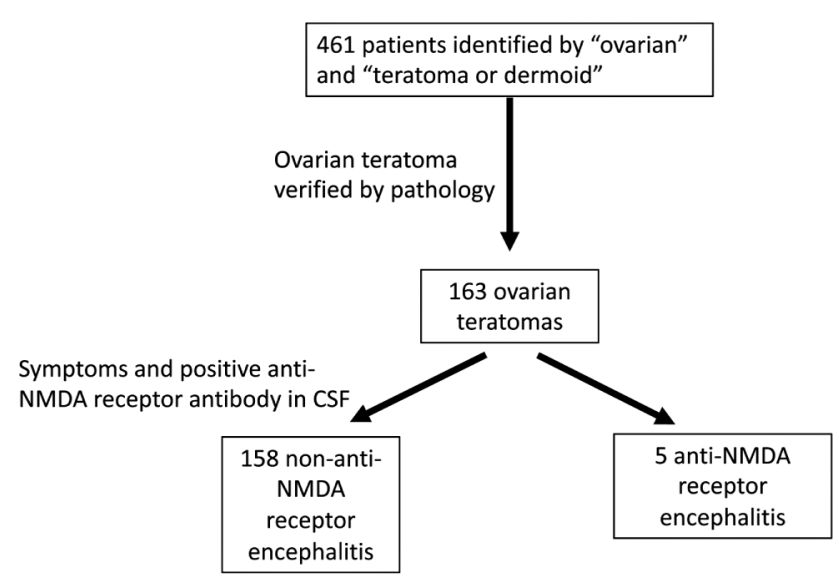

Fig. 1 Flow diagram of participants at each stage of the study. CSF, cerebrospinal fluid; NMDA, N-methyl-D-aspartate.

decreased level of consciousness; and (6) autonomic dysfunction or central hypoventilation ${ }^{5}$ (see - Fig. 1).

Descriptive summary statistics, Student's $t$-test, Fischer's exact test, and an analysis of variance (ANOVA) on a multiple linear regression model were performed using the R statistical software.

\section{Results}

Clinical characteristics are shown in - Table 1. Initial imaging methods to detect teratomas were ultrasound (70.55\%),

Table 1 Clinical characteristics in anti-NMDARE as compared with non-NMDARE patients

\begin{tabular}{|c|c|c|c|}
\hline & NMDARE $(n=5)$ & Non-NMDARE $(n=158)$ & $p$-Value \\
\hline Age, average years (SD) & $13.8(3.90)$ & $12.4(4.04)$ & 0.5 \\
\hline \multicolumn{4}{|l|}{ Race, $n(\%)$} \\
\hline African American & $4(80)$ & $67(41)$ & \\
\hline Caucasian & $1(20)$ & $55(34)$ & \\
\hline Latino & 0 & $24(15)$ & \\
\hline Asian & 0 & $10(6)$ & \\
\hline Native American & 0 & $1(0.6)$ & \\
\hline Mixed & 0 & $4(3)$ & \\
\hline Unknown & 0 & $2(1)$ & \\
\hline Ovarian teratoma volume, median $\mathrm{cm}^{3}$ (IQR) & $28.3(431.2)$ & $182.8(635.0)$ & 0.008 \\
\hline \multicolumn{4}{|l|}{ Ovarian teratoma pathology, $n(\%)$} \\
\hline Mature/benign & $5(100)$ & $150(95)$ & 1.0 \\
\hline Neuroglial element & $3(60)$ & $32(20)$ & 0.067 \\
\hline \multicolumn{4}{|l|}{ Neurological symptom, $n(\%)$} \\
\hline Psychiatric/cognitive decline & $5(100)$ & $0(0)$ & \\
\hline Speech dysfunction & $5(100)$ & $0(0)$ & \\
\hline Seizures & $3(60)$ & $0(0)$ & \\
\hline Movement disorder & $5(100)$ & $0(0)$ & \\
\hline Decreased level of consciousness & $2(40)$ & $0(0)$ & \\
\hline Autonomic dysfunction or central hypoventilation & $3(60)$ & $0(0)$ & \\
\hline
\end{tabular}

Abbreviations: IQR, interquartile range; NMDARE, N-methyl-D-aspartate receptor encephalitis; SD, standard deviation. 
computed tomographic scan (26.4\%), and magnetic resonance imaging (3.0\%). Five of 163 (3.1\%) patients with confirmed ovarian teratomas on pathology were diagnosed with NMDARE with the presence of at least one neurological symptom along with positive anti-NMDA receptor antibody detected in the CSF. Student's $t$-test demonstrated no difference in the ages of NMDARE versus non-NMDARE patients. All five NMDARE patients and 151/158 non-NMDARE patients had ovarian teratomas with benign pathology. Three of five (60\%) NMDARE had teratomas with identifiable neuroglial elements as compared with 32/158 (20.3\%) nonNMDARE patients, which trended toward significance (Fisher's exact test, $p=0.067$ ). For neurological symptoms, zero of the non-NMDARE patients reported any of the neurological symptoms associated with anti-NMDARE. For the NMDARE patients, all five patients exhibited psychiatric symptoms, speech dysfunction, and a form of movement disorder. A subset of NMDARE patients had one or more additional neurological symptoms, that is, seizures, decreased level of consciousness, and autonomic dysfunction/central hypoventilation ( $\mathbf{- T a b l e ~} \mathbf{1}$ ).

Using ANOVA, we evaluated predictors of ovarian teratoma volumes. Age had a positive correlation with teratoma size $(p=0.013)$, whereas NMDARE had an inverse correlation ( $p=0.008$, adjusted for age and race). Teratoma volumes from NMDARE patients were smaller than that of nonNMDARE patients (median $28.3 \mathrm{~cm}^{3}$ with interquartile range [IQR] of 431.2 and median 182.8 with IQR of 635.0, respectively; $p=0.01$ ).

\section{Discussion}

In our cohort, $3.07 \%$ of patients with ovarian teratomas had NMDARE. Moreover, ovarian teratoma volumes are smaller in NMDARE compared with non-NMDARE patients and increased age associates with a larger teratoma size. Additionally, $80 \%$ of NMDARE patients were African American, in comparison to $42.4 \%$ of non-NMDARE patients in this study; prior studies have varied in rates of African American NMDARE patients but another larger NMDARE cohort is $67 \%{ }^{6}$

NMDARE pathogenesis can be a paraneoplastic response to the neural components in ovarian teratomas. ${ }^{2,7}$ Neuroglial elements are possibly more commonly detected in NMDARE versus non-NMDARE ovarian teratomas, as our data trended toward significance and as discussed in a small case-control study. ${ }^{8}$ We also found that teratoma volumes are smaller in NMDARE versus non-NMDARE, which is most likely due to earlier tumor detection, similar to how neuroblastomas are detected earlier in opsoclonus myoclonus ataxia syndrome ${ }^{9}$ due to a paraneoplastic response to the tumor.

Limitations of this study include the retrospective nature of this study at a single center. Another limitation is that CSF anti-NMDA receptor antibody testing was not available for all individuals as routine anti-NMDA receptor antibody testing is not performed in patients with ovarian teratomas. How- ever, none of the symptoms associated with NMDARE were present in the non-NMDARE group and positive antibody alone would not be sufficient for diagnosis. ${ }^{5}$ Serum antiNMDAR antibody testing is inadequate because anti-NMDAR antibody detection is more sensitive in $\mathrm{CSF}^{3}$ than serum and serum anti-NMDAR antibody testing can have false positives. $^{10}$

In conclusion, rates of NMDARE in ovarian teratomas are low (3.07\%) and ovarian teratomas are smaller in NMDARE than in non-NMDARE. Neuroglial elements may contribute to the development of NMDARE. Further studies are needed to understand the timing of anti-NMDA receptor antibodies in teratomas and development of NMDARE.

\section{Funding}

This work was not funded.

\section{Conflict of Interest}

Jennifer Li has no disclosures.

Sarah Milla has no disclosures.

Grace Gombolay receives salary support from the Centers for Disease Control and Prevention for the surveillance of acute flaccid myelitis.

\section{References}

1 Dalmau J, Gleichman AJ, Hughes EG, et al. Anti-NMDA-receptor encephalitis: case series and analysis of the effects of antibodies. Lancet Neurol 2008;7(12):1091-1098

2 Dalmau J, Tüzün E, Wu HY, et al. Paraneoplastic anti-N-methyl-Daspartate receptor encephalitis associated with ovarian teratoma. Ann Neurol 2007;61(01):25-36

3 Titulaer MJ, McCracken L, Gabilondo I, et al. Treatment and prognostic factors for long-term outcome in patients with antiNMDA receptor encephalitis: an observational cohort study. Lancet Neurol 2013;12(02):157-165

4 Chefdeville A, Treilleux I, Mayeur ME, et al. Immunopathological characterization of ovarian teratomas associated with anti-Nmethyl-D-aspartate receptor encephalitis. Acta Neuropathol Commun 2019;7(01):38. Doi: 10.1186/s40478-019-0693-7

5 Graus F, Titulaer MJ, Balu R, et al. A clinical approach to diagnosis of autoimmune encephalitis. Lancet Neurol 2016;15(04): 391-404

6 Howarth RA, Vova J, Blackwell LS. Early functional outcomes for pediatric patients diagnosed with anti-NMDA receptor encephalitis during inpatient rehabilitation. Am J Phys Med Rehabil 2018; 30(Oct):-・. Doi: 10.1097/PHM.0000000000001087

7 Day GS, Laiq S, Tang-Wai DF, Munoz DG. Abnormal neurons in teratomas in NMDAR encephalitis. JAMA Neurol 2014;71(06): 717-724

8 Chiu HC, Su YC, Huang SC, Chiang HL, Huang PS. Anti-NMDAR encephalitis with ovarian teratomas: review of the literature and two case reports. Taiwan J Obstet Gynecol 2019;58(03):313-317

9 Rudnick E, Khakoo Y, Antunes NL, et al. Opsoclonus-myoclonusataxia syndrome in neuroblastoma: clinical outcome and antineuronal antibodies-a report from the Children's Cancer Group Study. Med Pediatr Oncol 2001;36(06):612-622

10 Dahm L, Ott C, Steiner J, et al. Seroprevalence of autoantibodies against brain antigens in health and disease. Ann Neurol 2014;76 (01):82-94 